\title{
A novel method for distinguishing the intersegmental plane: pulmonary circulation single-blocking
}

\author{
Min Zhang ${ }^{1}$, Ning $\mathrm{Mao}^{2}$, Qing-Chen Wu ${ }^{1}$, Ming-Jian Ge ${ }^{1}$ \\ ${ }^{1}$ Department of Cardiothoracic Surgery, The First Affiliated Hospital of Chongqing Medical University, Chongqing, China; ${ }^{2}$ Department of \\ Cardiothoracic Surgery, Yongchuan Hospital of Chongqing Medical University, Chongqing, China \\ Correspondence to: Ming-Jian Ge. Department of Cardiothoracic Surgery, The First Affiliated Hospital of Chongqing Medical University, Chongqing \\ 400016, China. Email: 253616309@qq.com.
}

Submitted Nov 06, 2020. Accepted for publication Dec 26, 2020.

doi: $10.21037 /$ jtd-20-3428

View this article at: http://dx.doi.org/10.21037/jtd-20-3428

\section{Introduction}

Ground-glass opacity (GGO) is a common radiological manifestation in East Asian countries, such as China and Japan. Besides benign lesions, GGOs can be a specific type of lung adenocarcinomas or their preinvasive lesions. The GGO that received VATS has a high proportion of malignant, its prognosis is satisfied. For these lesions, especially those with diameters of less than $2 \mathrm{~cm}$ and a consolidation tumor ratio (CTR) of less than $50 \%$, segmentectomy is recommended. Video-assisted thoracoscopic surgery (VATS) segmentectomy is technically difficult. The identification of the intersegmental plane is considered the most difficult step of segmentectomy. The traditional inflation-deflation method used to visualize the intersegmental plane requires the double-blocking of blood circulation (in both the artery and the vein) of the target segment. In this paper, we present a series of cases of segmentectomies in which a novel method (i.e., the pulmonary circulation single-blocking method) was used to identify the intersegmental plane. This modified technique combined the guidance of preoperative three-dimensional computed tomography bronchography and angiography (3D-CTBA) and intraoperative discernment without any additional auxiliary materials.

\section{Operative techniques}

From January 2019 to March 2020, 83 patients underwent anatomic segmentectomies in which the pulmonary circulation single-blocking method was used (see Table 1). Before each operation, all the CT images were reconstructed to evaluate the variation of anatomy. 3D-CTBA was used to identify the dominant pulmonary artery supplying the target segment, and the intrasegmental vein collecting the target segment. During each operation, the dominant pulmonary artery or intrasegmental vein of the target segment was first ligated. The lung was then ventilated using the traditional inflation-deflation method. The segmentectomy was then performed after the intersegmental demarcation had been confirmed. All procedures performed in this study involving human participants were in accordance with the Declaration of Helsinki. The study was approved by ethics board of our hospital (No. 2020-206) and informed consent was taken from all the patients.

\section{Pulmonary vein single-blocking: $R S 3$}

A 40-year-old female patient was admitted to our hospital for a lesion in RS3. A high-resolution computed tomography (HRCT) scan revealed a 20-mm GGO. Preoperative 3D-CTBA images identified the intrasegmental veins as $\mathrm{V} 3 \mathrm{a}, \mathrm{V} 3 \mathrm{~b}$ and $\mathrm{V} 3 \mathrm{c}$, and the intersegmental veins as V2c and $\mathrm{V} 1 \mathrm{~b}$, and showed that $\mathrm{A} 3$ ran along $\mathrm{B} 3$. The patient was placed in the left lateral decubitus position with the main surgeon at the ventral side. Under general anesthesia and single-lung ventilation, the main utility incision and observing port was made in the fourth and seventh intercostal space in the anterior axillary line. Two assistant 
Table 1 Summary of operative procedures

\begin{tabular}{|c|c|c|c|}
\hline Group & SVG & SAG & $P$ value \\
\hline \multicolumn{4}{|l|}{ Segmentectomy } \\
\hline Right S1 & 0 & 9 & \\
\hline Right S2 & 4 & 3 & \\
\hline Right S3 & 5 & 0 & \\
\hline Right S4 & 6 & 0 & \\
\hline Right S5 & 2 & 0 & \\
\hline Right S6 & 0 & 6 & \\
\hline Right S7 & 0 & 2 & \\
\hline Right S9+10 & 1 & 1 & \\
\hline Right $\mathrm{S} 2 \mathrm{~b}+\mathrm{S} 3 \mathrm{a}$ & 4 & 0 & \\
\hline Right $S 6 b+S 8 a$ & 0 & 3 & \\
\hline Left S1+2 & 0 & 8 & \\
\hline Left $\mathrm{S} 1+2 \mathrm{c}+\mathrm{S} 3 \mathrm{a}$ & 0 & 3 & \\
\hline Left $\mathrm{S} 1+2 \mathrm{a}+\mathrm{S} 3 \mathrm{c}$ & 2 & 0 & \\
\hline Left S3 & 7 & 0 & \\
\hline Left S6 & 0 & 3 & \\
\hline Left S8 & 0 & 5 & \\
\hline Left S9+10 & 0 & 6 & \\
\hline Left S9a & 0 & 3 & \\
\hline Division of segmental plane & & & 0.823 \\
\hline Staple & 0 & 0 & \\
\hline Cautery & 0 & 2 (RS7) & \\
\hline Staple and cautery & 31 & 50 & \\
\hline Operating time (min) & $65 \pm 9$ & $58 \pm 6$ & 0.786 \\
\hline Blood loss (mL) & $123 \pm 15$ & $143 \pm 12$ & 0.775 \\
\hline Duration of drainage (d) & $2.2 \pm 0.8$ & $3.1 \pm 0.6$ & 0.659 \\
\hline Length of stay (d) & $3.1 \pm 1.2$ & $3.3 \pm 0.9$ & 0.824 \\
\hline Complications & & & 1 \\
\hline Prolonged air leak & 0 & 0 & \\
\hline Postoperative bleeding & 0 & 0 & \\
\hline
\end{tabular}

SVG, singe vein group; SAG, single artery group.

incisions were made in the seventh intercostal space in the mid-axillary line and the ninth intercostal space in the posterior axillary line. The V3a, V3b, and V3c were first divided and resected. The anesthesiologist then ventilated the lung using $100 \%$ oxygen. During the 10 -minute period in which we waited for the visualization of the intersegmental plane, the target bronchus (B3) and artery (A3) were dissected and resected. The intersegmental plane was dissected using electrocautery and a stapler along the demarcation line. The dissected plane was not covered with anything (see Video 1).

\section{Pulmonary artery single-blocking: RS8a}

A 50-year-old female patient was admitted to our hospital for a lesion in the right lower lobe (RLL). A HRCT scan revealed an 8-mm GGO. Preoperative 3D-CTBA images showed a lesion located in S8a, and that A8a went through the lesion. The patient was placed in the right lateral decubitus position (the locations of the ports were the same as those described above). The A8a was first divided and resected. The target bronchus (i.e., B8a) was not dissected. The anesthesiologist then ventilated the lung. After the 10 -minute period in which we waited for the visualization of the intersegmental plane, the intersegmental plane was dissected using a stapler (see Video 2).

\section{Comments}

The visualization of the intersegmental plane is one of the core techniques of the VATS segmentectomy. Compared with other methods, such as bronchofiberoptic jet ventilation and the intravenous injection of indocyanine green, the inflation-deflation method requires no special instrument and has no risk of allergic reaction. It is the most commonly used method in China. In relation to the conventional inflation-deflation method, the bronchus of the target segment is first clamped and the lung is then ventilated. A poor line develops when this method is used, as the positive pressure used to restore inflation is transmitted not only to the preserved segments but also to the target segment by means of the collateral ventilation through Cohn's pores (1). Noriaki improved this method by first ventilating the lung and then clamping the bronchus of the target segment. Under this method, the bronchus of the preserved segments is left open under atmospheric pressure, which allows the gas inside the preserved segments to escape due to a recoiling of the elastic fibers. It is believed that there is no chance that the Cohn's pores will open when this method is used (2). Hisashi Iwata innovatively modified the inflation-deflation method by taking the factor 
of gas exchange into consideration. Under this method, not only is the target segmental bronchus cut, but so is the artery and vein. The entire lobe is then inflated with pure oxygen. The alveoli in which the pulmonary circulation continues (in the preserved segments) can perform gas exchange and absorb oxygen (in the hypoxia zone). Alveoli with no pulmonary circulation (including those in the target segments in which both the artery and vein are cut) are not involved in gas exchange and retain oxygen inside (in the hyperoxia zone). The border between the hyperoxia zone (the inflation area) and the hypoxia zone (the deflation area) defines the intersegmental plane (3).

We made a small modification to our pulmonary circulation single-blocking method. As the pulmonary circulation has two decisive factors (i.e., the artery and the vein), the cut of either an artery or vein could result in an interruption to pulmonary circulation. Based on this theory, we cut the target artery (vein) first, and the entire lobe was then inflated immediately. During the waiting period for the intersegmental plane, the target bronchus and as the target vein (artery) were dissected and resected.

In relation to cases in which the GGO is located very peripherally, the resection of the target bronchus can also be ignored. This method has a number of advantages. First, it is easy to learn and use and does not require any special instruments. Second, it can save time, as the target bronchus is dissected and resected during the period spent waiting for the visualization of the intersegmental plane. Third, as the bronchus is not resected at the moment of inflation, it is easy to achieve full re-expansion with relatively low pressure. This could reduce any pressure-related damage to the lung caused by manual ventilation. Fourth, under the traditional inflation-deflation method, the structures (bronchus and vessels) of the target segment are all resected until the moment at which the intersegmental plane is visualized; thus, mistakes cannot be corrected. Conversely, under the single-blocking method, not all of the structures of the target segment are resected before the moment at which the intersegmental plane is visualized. Thus, there is a second opportunity to identify the target segment structures (the first opportunity to do so is using the preoperative 3D-CTBA scans). This second opportunity enables the structures that run into the inflation area (the hyperoxia zone) to be cut and those that run into the deflation area (the hypoxia zone) to be preserved. We call this the "doublecheck." The combination of the preoperative 3D-CTBA and the intraoperative discerning "double-check" effectively ensures the accurate identification of the target structures.

In conclusion, this study showed the feasibility and safety of a thoracoscopic segmentectomy using the pulmonary circulation single-blocking method. This method could simplify the traditional inflation-deflation method. Further, the use of the "double-check" could ensure that mistakes are avoided in the identification of target structures.

\section{Acknowledgments}

Funding: This work was supported by the Natural Science Foundation of Yongchuan Science and Technology Committee, Chongqing, China (Ycstc, 2017nc5008).

\section{Footnote}

Conflicts of Interest: All authors have completed the ICMJE uniform disclosure form (available at http://dx.doi. org/10.21037/jtd-20-3428). The authors have no conflicts of interest to declare.

Ethical Statement: The authors are accountable for all aspects of the work in ensuring that questions related to the accuracy or integrity of any part of the work are appropriately investigated and resolved. All procedures performed in this study involving human participants were in accordance with the Declaration of Helsinki (as revised in 2013). The study was approved by ethics board of our hospital (No. 2020-206) and informed consent was taken from all the patients.

Open Access Statement: This is an Open Access article distributed in accordance with the Creative Commons Attribution-NonCommercial-NoDerivs 4.0 International License (CC BY-NC-ND 4.0), which permits the noncommercial replication and distribution of the article with the strict proviso that no changes or edits are made and the original work is properly cited (including links to both the formal publication through the relevant DOI and the license). See: https://creativecommons.org/licenses/by-nc-nd/4.0/.

\section{References}

1. Shields TW. General thoracic surgery, 2 nd edn. Lea and Febiger, Philadelphia, 1983:326-38. 
2. Yajima T, Shimizu K, Mogi A, et al. Thoracoscopic right middle lobe segmentectomy. Gen Thorac Cardiovasc Surg 2019;67:344-7.

Cite this article as: Zhang M, Mao N, Wu QC, Ge MJ. A novel method for distinguishing the intersegmental plane: pulmonary circulation single-blocking. J Thorac Dis 2021;13(1):362-365. doi: 10.21037/jtd-20-3428
3. Iwata H, Shirahashi K, Mizuno Y, et al. Surgical technique of lung segmental resection with two intersegmental planes. Interact Cardiovasc Thorac Surg 2013;16:423-5. 